\title{
Correction to: Representation of light pressure resultant force and moment as a tensor series
}

\author{
Nikolay Nerovny ${ }^{1}$ (D) Vladimir Zimin ${ }^{1}$. \\ Sergey Fedorchuk ${ }^{2}$. Evgeny Golubev ${ }^{2}$
}

Published online: 26 October 2017

(C) Springer Science+Business Media B.V. 2017

\section{Correction to: Celest Mech Dyn Astr (2017) 128:483-513 DOI 10.1007/s10569-017-9758-8}

In the paper Nerovny et al. (2017), the commentaries about a convergence of series which represent the absolute value function and corresponding equations contain several mistakes (Sect. 2, from Eqs. (4) to (6)).

The series Eq. (3)

$$
|\hat{\mathbf{n}} \cdot \hat{\mathbf{s}}|=|x|=\frac{2}{\pi}-\frac{4}{\pi} \sum_{n=1}^{\infty} \frac{(-1)^{n} T_{2 n}(x)}{-1+4 n^{2}}
$$

of Chebyshev polynomials of the first kind for $|\hat{\mathbf{n}} \cdot \hat{\mathbf{s}}|=|x| \leq 1$ is absolutely convergent. If we define $x=\cos y$, than $T_{2 n}=\cos 2 n y,\left|T_{2 n}\right| \leq 1$, and we get the ordinary Fourier series

The online version of the original article can be found under doi:10.1007/s10569-017-9758-8.

$凶$ Nikolay Nerovny

nick.nerovny@bmstu.ru

Vladimir Zimin

zimin@bmstu.ru

Sergey Fedorchuk

fedorchuk@asc.rssi.ru

Evgeny Golubev

golubev.ev@asc.rssi.ru

1 Bauman Moscow State Technical University, 5 stroenie 1, 2-ya Baumanskaya St., Moscow, Russian Federation 105005

2 Astro Space Center of P.N. Lebedev Physical Institute, 84/32 Profsoyuznaya St., GSP-7, Moscow, Russian Federation 117997 
which is majorizable by the following convergent series:

$$
\frac{2}{\pi}-\frac{4}{\pi} \sum_{n=1}^{\infty} \frac{1}{-1+4 n^{2}} .
$$

Additionally, for any $x$ the original series is an alternating Leibniz series. Its partial sum differs from $|x|$ less or equal than the absolute value of the first neglected term.

These are the steps to produce a power series of absolute value function from Eq. (3):

$$
\begin{aligned}
|\hat{\mathbf{n}} \cdot \hat{\mathbf{s}}| & =-\lim _{N_{\max } \rightarrow \infty} \frac{4}{\pi} \sum_{n=1}^{N_{\max }} \sum_{k=0}^{n-1} \frac{(-1)^{n}(-1)^{k} n(2 n-k-1) !}{\left(-1+4 n^{2}\right) k !(2 n-2 k) !} 4^{n-k}(\hat{\mathbf{n}} \cdot \hat{\mathbf{s}})^{2(n-k)}= \\
& (\text { let } m=n-k) \\
& =-\lim _{N_{\max } \rightarrow \infty} \sum_{m=1}^{N_{\max }} \frac{(-1)^{m} 4^{m+1}}{\pi(2 m) !} \sum_{n=m}^{N_{\max }} \frac{n(n+m-1) !}{\left(-1+4 n^{2}\right)(n-m) !}(\hat{\mathbf{n}} \cdot \hat{\mathbf{s}})^{2 m} .
\end{aligned}
$$

That's why the Eqs. (4) and (5) from Nerovny et al. (2017) should be written as follows:

$$
\begin{aligned}
|\hat{\mathbf{n}} \cdot \hat{\mathbf{s}}| & =\lim _{N_{\max } \rightarrow \infty} \sum_{m=1}^{N_{\max }} B_{m}(\hat{\mathbf{n}} \cdot \hat{\mathbf{s}})^{2 m} \approx \sum_{m=1}^{N_{\max }} B_{m}(\hat{\mathbf{n}} \cdot \hat{\mathbf{s}})^{2 m}, \\
B_{m} & \approx-\frac{(-1)^{m} 4^{m+1}}{\pi(2 m) !} \sum_{n=m}^{N_{\max }} \frac{n(n+m-1) !}{\left(-1+4 n^{2}\right)(n-m) !},
\end{aligned}
$$

and in equations for $N_{\max B}$, Eqs. (6) and (34), the $\left\lfloor\left(N_{\max }-1\right) / 2\right\rfloor$ term should be replaced by $N_{\max }$.

The results of calculations in Sects. 7 and 8 are not affected by this error because in the numerical calculations we used correct relations presented in this erratum.

\section{Reference}

Nerovny, N., Zimin, V., Fedorchuk, S., Golubev, E.: Representation of light pressure resultant force and moment as a tensor series. Celest. Mech. Dyn. Astron. 128, 483-513 (2017). doi:10.1007/s10569-0179758-8 